\title{
The teenage coeliac: follow up study of 102 patients
}

\author{
P J KUMAR, ${ }^{*}$ J WALKER-SMITH, $\dagger$ P MILLA,$\ddagger$ G HARRIS,${ }^{*}$ J COLYER,${ }^{*}$ AND R HALLIDAY* \\ ${ }^{*}$ Department of Gastroenterology, St Bartholomew's Hospital, †Queen Elizabeth Hospital for Children, \\ Hackney Road, and $¥$ Hospital for Sick Children, London
}

SUMMARY Over a 10 year period a total of 102 teenage patients with coeliac disease were assessed on transfer from paediatric hospitals to an adult clinic. Fifty seven patients said they were on a strict gluten free diet; 36 were semistrict, and nine admitted to eating a normal diet. Jejunal mucosal abnormalities, however, suggested that many patients on the 'strict' diet were actually consuming gluten. All patients were well with biochemical parameters within the normal range. Height percentiles were not significantly different from the normal population but patients, as a group, were significantly lighter.

The clinical presentation of coeliac disease in childhood and adult life has been well documented. Little is known, however, about the progress of children with coeliac disease in the 'teenage' years. Three previous studies have assessed the wellbeing of patients after growth into young adult life. ${ }^{1-3} \mathrm{On}$ the basis of clinical and laboratory findings, these groups came to the conclusion that a strict gluten free diet should be recommended for life. Compliance with this strict diet is, however, very variable. ${ }^{45}$

The aim of this study was twofold. Firstly, we wished to assess the general wellbeing of teenage patients with coeliac disease and to correlate this with the strictness of their gluten free diet. Secondly, we wondered whether the feeding patterns, ethnic origins, or age at diagnosis had changed from those previously quoted for children with coeliac disease.

\section{Patients and methods}

A total of 102 patients (56 females, 46 males) aged 12-20 years with coeliac disease were assessed in the coeliac clinic during the years 1974-84. Most of the patients were referred for their continued follow up in adult life by two centres: 47 patients came from the Hospital for Sick Children and 38 from Queen Elizabeth Hospital for Children; 17 patients came from other centres or from general practitioners.

At transfer all patients were seen by one of us (PK). All had a detailed case history taken. A clinical examination was performed with the documentation of their height and weight on percentile charts. All patients were offered blood tests but some refused on the basis that they felt very well. Most patients, however, had a full blood count, liver function tests, and measurements of serum $B_{12}$ and red cell folate performed. Patients were asked to assess their own diet as strict (no gluten intake), semistrict (consuming several items of gluten per week), or normal (no gluten restriction at all); in some patients a formal dietary check was conducted by a dietitian.

Patients were offered a jejunal biopsy either for a gluten challenge (if this had not been performed) or if a biopsy had not been performed for two years or more. Forty four patients agreed to have a jejunal biopsy. Biopsies were taken at the duodenojejunal junction under fluoroscopic control using a Crosby capsule. Villous heights were measured using an eye piece micrometer. An average count of 10 villi on each specimen was made under $\times 10$ magnification.

\section{Results}

All patients were well and leading normal healthy lives. One patient, however, although otherwise well, had symptoms of the irritable bowel syndrome that were bad enough to necessitate further radiological studies of her bowel. There were no other symptoms referable to the gastrointestinal tract apart from the 'occasional' attack of diarrhoea or abdominal pain which was passed off, by parents and patients alike, as part of normal life.

The patients' assessment of the strictness of their diet before transfer to an adult clinic was as follows: those who considered their diet to be strict, 57; semistrict, 36; and normal, nine. After follow up in an adult clinic for more than one year the numbers 
were: strict, 45; semistrict, 46; and normal, 11 . The gluten equivalent (assessed by the dietitian) was: strict, no gluten; semistrict, 2.5-10 g gluten; and normal, $>10 \mathrm{~g}$ gluten. In a few cases the actual diet eaten by patients was only admitted to after the parents had left the consulting room.

Although most patients were very well informed about their diets a few children, although ostensibly on a strict diet, had no idea about the gluten content of various foods when repeatedly questioned (with their mothers) by the dietitian. Nevertheless, there was a good correlation $(r=0 \cdot 888)$ and no significant difference $(p=>0.22)$ between the dietitian and patients own view of the strictness of the diet. This was not surprising as the former could only assess the diet on the information given by the patient. Most patients appeared to have self-selected their diets; almost all patients on the 'strict' diet found it no problem while those on the normal diet found a strict diet difficult to adhere to for practical or social reasons. (For example, the marriage prospects for an Asian girl were reduced if she was known to be on a diet). In some cases, although patients said they had gluten free school lunches and gluten free food at home, when they went out with their friends at 'break-time' they ate hamburgers, rolls, biscuits, cakes, etc.

Patients appeared not to have any major problem over having gluten free food at school; most patients took gluten free packed lunches while some schools actually provided gluten free meals. One patient at boarding school had a term's worth of gluten free bread stored in a freezer at school.

The assessment of the diet after follow up in an adult clinic for more than one year (see above) was made to see if the transfer from a paediatric to an adult centre made children less conscientious over their diets. Only a few teenagers had become less strict during their follow up in an adult clinic. It should be emphasised that all children were on regular follow up and the policy in both paediatric and adult centres was to advise patients to adhere to a strict gluten free diet.

Of the ethnic origins of the patients, 23 had first degree relatives with Irish origins. The remainder were white but not Irish; two were of Asian origin.

When the feeding patterns in infancy were examined, 36 of 69 patients were breast fed. The weaning age varied from 3 weeks to 1 year of age with an average of 3.76 months.

Fifty one patients were diagnosed in the first 2 years of life and 26 over 10 years of age. Symptoms were noticed from a few weeks to many months after weaning (mean $=2.8$ months). There was a delay in diagnosis from the onset of symptoms of up to 14 years, with 34 patients being diagnosed within two months of onset of symptoms, but 12 had had symptoms for five years or more. There was no correlation between the actual age of weaning and the presentation of symptoms in those patients diagnosed at an older age.

Associated and past diseases are shown in the table. Twelve of the 102 patients had atopic illnesses: asthma $(n=3)$, eczema $(n=6)$, and hayfever $(n=3)$. This incidence is the same as that seen in the general population and there was no correlation with the feeding patterns in infancy. Twenty six patients had first degree relatives with atopic illnesses and of these, 10 had more than one first degree relative with atopy.

PERCENTILES AT TRANSFER TO ST BARTHOLOMEW'S HOSPITAL

There were eight patients out of 87 who were below the 3rd percentile for weight (five female, three male) and six of these were on 'strict' gluten free diets. Of these six, three had presented over the age of 10 years with shortness of stature; one patient had developed thyrotoxicosis and one had diabetes mellitus and asthma, but had reached puberty at transfer. One further patient reached puberty within a few months of transfer. Analyses and comparison with the general population distribution showed that all patients as a group were significantly lighter $(p<0.01$; see fig 1$)$.

There were six out of 70 patients who were below the 3rd centile for height (four female, two male); five of these were on a strict gluten free diet. Again three patients were those who had presented over the age of 10 years with shortness in stature and one patient had diabetes mellitus and asthma. Five had

Table No of associated and past illnesses in teenagers with coeliac disease

\begin{tabular}{ll}
\hline Disease & No \\
\hline Thyroid disease & 2 \\
Diabetes mellitus & 1 \\
Rheumatoid arthritis & 1 \\
Glomerular nephritis + chronic active hepatitis & 1 \\
Idiopathic pulmonary haemosiderosis & 1 \\
Sacroileitis & 1 \\
Non-specific joint pains & 3 \\
Asthma & 3 \\
Ezcema & 6 \\
Hay fever & 3 \\
Undescended testes & 2 \\
Pyloric stenosis & 1 \\
Epilepsy & 1 \\
Renal colic & 1 \\
Psoriasis & 2 \\
Pancreatic insufficiency & 1 \\
Hernias & 4 \\
\hline
\end{tabular}


reached puberty at transfer. On completion of growth all six remained below the 3 rd centile. There was no significant difference in height centiles compared with the normal population distributions $(\mathrm{p}=>0 \cdot 22$; fig 2$)$.

\section{LABORATORY DATA}

\section{Haematology}

Three out of 95 patients (all female) had haemoglobin concentrations that were less than the lower range of normal females $(115 \mathrm{~g} / \mathrm{l})$. Two of these patients were on a 'strict' diet. All three had started their periods but had no history of menorrhagia. Six out of 73 had abnormal red cell folate concentrations that were below $363 \mathrm{nmol} / \mathrm{l}$ : one with a concentration of $265 \mathrm{nmol} / 1$ and the remaining five between $283-333 \mathrm{nmol} / \mathrm{l}$; two of these patients stated they were on a 'strict' gluten free diet.

One of 81 patients had a serum $B_{12}$ concentration of $107 \mathrm{pmol} / 1$ (normal $>150 \mathrm{pmol} / \mathrm{l})$. This patient admitted to occasional gluten intake. A whole body $\mathrm{B}_{12}$ absorption test showed a normal absorption of $80 \%$ in this patient. No cause was found for the low

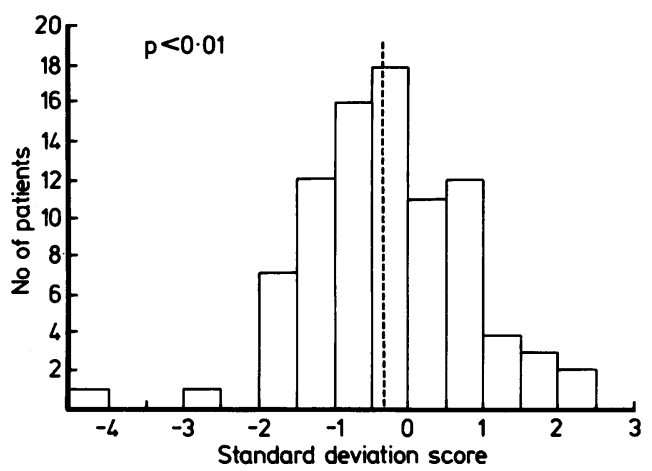

Fig 1 Weight percentiles at transfer to adult clinic in 87 patients. The dotted line is the median.

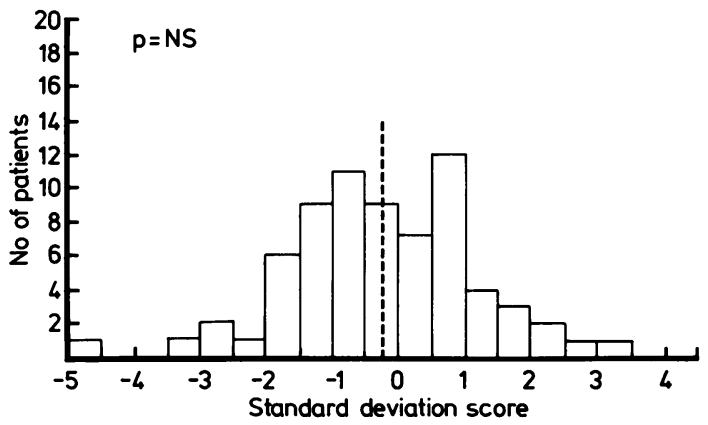

Fig 2 Height percentiles at transfer to adult clinic in 70 patients. The dotted line is the median. serum $\mathrm{B}_{12}$ concentration; this was repeated a year later and was within the normal range.

\section{Biochemistry}

Serum protein and calcium concentrations were within the normal range of our laboratories in 80 patients. One patient had an albumin of $3.4 \mathrm{~g} / 1$ (normal $>3.5 \mathrm{~g} / \mathrm{l}$ ), and although she said she was on a 'strict' diet, the jejunal biopsy specimen was flat. Alkaline phosphatase concentrations were variable and raised above the normal of $120 \mathrm{IU} / \mathrm{l}$ in 26 of 78 patients. This was compatible with their age and growth.

\section{Appearance of jejunal mucosa}

The villus heights of 44 patients are shown in fig 3 . Seventeen patients had gross abnormality of villus heights of below $200 \mu \mathrm{m}$; of these five claimed to be on a strict gluten free diet and seven were on a semistrict diet. All patients on normal diets had abnormal biopsy specimens.

\section{Discussion}

This study shows that all teenagers with coeliac

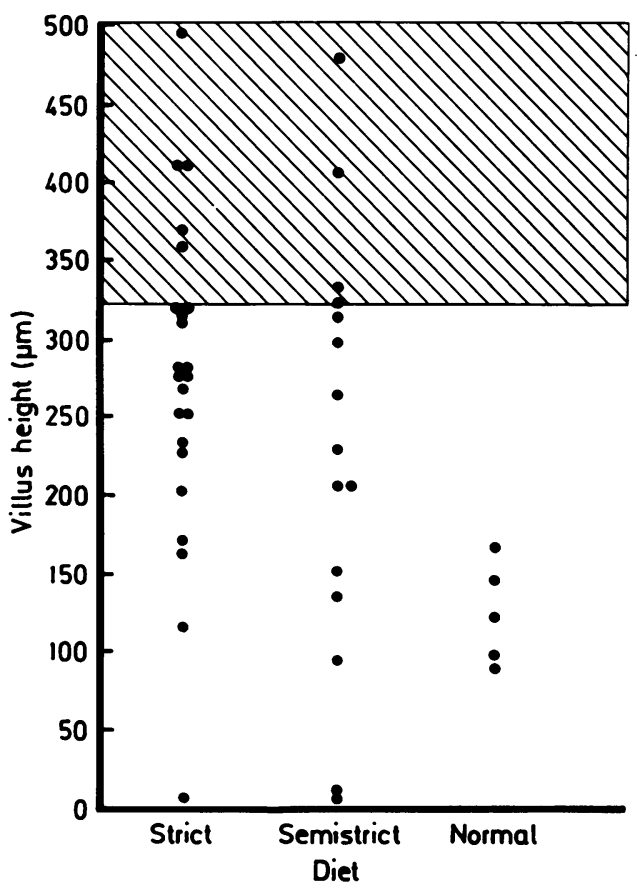

Fig 3 Villus heights of jejunal mucosa in 44 patients at transfer to adult clinic. Patients are divided into groups on a strict gluten free diet, a semistrict diet and those on a normal diet. The hatched area shows the normal range. 
disease were clinically asymptomatic and leading normal healthy lives. Only 57 of the patients, however, were keeping to a strict gluten free diet and nine even admitted to being on a normal diet. Abnormalities of the jejunal mucosa, however, suggested that many more patients were consuming gluten despite being on a 'strict' diet. Although it is possible that these abnormalities of villous height might have been due to a factor other than gluten, four of the five patients in the strict group previously had had a positive gluten challenge (that is, jejunal mucosal deterioration on gluten reintroduction). Laboratory data showed a few abnormalities but all of these were minimal. The red cell folate concentration, measured by the microbiological method, is usually considered to be a good index of untreated coeliac disease. In this series only six of the 73 patients tested had low red cell folate concentrations, suggesting that 'the low gluten diet' that these patients were consuming caused mucosal abnormalities that were insufficient to affect the absorption of nutrients. In contrast, Weir and Hourihane found the concentration of serum folate was the best single measure of whether a patient was maintaining a strict gluten diet, but the number of patients reported were small. ${ }^{5}$

Our results are in accord with Jackson et al who found that $40 \%$ of 50 children were not on a strict gluten free diet. ${ }^{4}$ Formal gluten challenge studies have shown that patients often do not develop symptoms on the reintroduction of gluten. Six of 27 children were asymptomatic in a study by WalkerSmith et $a l,{ }^{6}$ and two thirds of teenage and adult patients also remained well, despite jejunal morphological deterioration in another study using long term gluten challenge. ${ }^{7}$ Visakorpi showed that it may take many years for children to relapse. ${ }^{8}$

Analysis of the height and weight percentiles showed that although there was no significant difference in height when compared with the normal population distribution, patients with coeliac disease were significantly lighter than their age and sex matched controls. However, we were unable to correlate the strictness of their diet with either the height or the weight centiles that they achieved. Three patients had presented with shortness of stature and were not diagnosed until after the age of 10 years-that is, they were too old for catch up growth to occur.

There was no difference in the feeding patterns in infancy of patients when compared with those seen in the general population. The patterns of breast feeding and weaning age were the same as those seen in the general population in the 1960s and 1970s. This suggests that feeding patterns in infancy had no effect on the presentation of coeliac disease.
The age at diagnosis of our patients was very variable. Sixty one patients were diagnosed in the first 2 years of life, but 26 were diagnosed at 10 years and over. Although those diagnosed in the first 2 years became symptomatic soon after weaning there was no correlation between the age of weaning and the presentation of the symptoms in those diagnosed at an older age. About $50 \%$ of this older group had, in fact, first presented to the adult clinic. The delay in diagnosis was again extremely variable but a third of patients were diagnosed within two months of the onset of their symptoms.

The associated and past diseases showed no increased incidence of any particular condition. We could not relate our atopic patients with patterns in breast feeding or bottle feeding, but the numbers are small.

There have been four previous studies documenting the follow up of patients originally diagnosed in childhood. Mortimer et al followed up 10 adult patients in whom the diagnosis had been made in childhood 18 to 38 years earlier. ${ }^{3}$ Nine had a flattened jejunal mucosa but despite this two were asymptomatic. Sheldon in 1969 followed 57 patients (aged 16-27 years) at the Hospital for Sick Children who were originally diagnosed in childhood and treated with two year courses of gluten free diet. ${ }^{1}$ $\mathrm{He}$ found that on a normal diet 13 patients had relapsed but 44 patients remained extremely well. Of these 44, 19 had low serum folate concentrations, 11 had low serum iron, and six were short. On the basis of this he suggested that a lifelong gluten free diet should be the treatment for these patients. Two years later Young and Pringle, at Queen Elizabeth Hospital for Children, followed up 44 patients diagnosed in childhood after eight to 19 years. ${ }^{2}$ The 20 patients on a gluten free diet were well; however, of the 24 on a normal diet only five were well. The remaining 19 of these patients had 'diarrhoea relapses' and five were anaemic. Again these authors suggested that a lifelong gluten free diet should be recommended treatment for coeliac disease. Weir and Hourihane followed up 10 patients for four to six years; five patients did not persist with a gluten free diet and although their jejunal biopsies were abnormal, they had no abnormalities on laboratory investigation. ${ }^{5}$

There is no doubt that a gluten free diet is beneficial to patients with coeliac disease. Despite a policy of advocating a strict gluten free diet with regular checks at outpatient follow up, however, our study has shown that many of our patients were not strictly adhering to a gluten free diet, yet all were asymptomatic. Recently, the concept of the permanence of gluten sensitivity in patients with coeliac disease has been challenged. Schmitz et al have 
continued a normal diet in children with confirmed coeliac disease and have shown that the jejunal mucosa actually improved on this diet. ${ }^{9}$ Shmerling and Franckx delineated a group $(6 \cdot 6 \%)$ of their patients who did not relapse and showed no alteration of the intestinal mucosa after stopping their gluten free diet for at least 2.4 years. ${ }^{10}$ These studies put the current definition of coeliac disease into question.

In our study patients remained well despite gluten ingestion. As they were lighter in weight as a group, however, it could be argued that they should keep to a strict gluten free diet. Nevertheless, many patients will 'cheat' and will continue consuming gluten in their diets. It is possible that the actual amount of gluten ingested may be the relevant factor in producing symptoms, whereby small amounts are well tolerated. The fact that children and adolescents may take much longer to relapse has been shown in previous studies ${ }^{7811}$ and it is possible that some of our patients may develop symptoms in the future. We would therefore recommend that these patients are followed up regularly with blood tests to detect occult nutritional deficiencies.

We would like to thank Mr Peter Davies of the growth and development department at the Hospital for Sick Children for analyses of percentiles; the late Professor John Harries for referral of patients from the Hospital for Sick Children; Dr AM Dawson for investigating patients under his care; Miss Jasmine Challis of the dietetic department at St Bartholomew's Hospital; the Joint Research Board of The City and Hackney Health Authority, and the Coeliac Society for their financial help.

\section{References}

1 Sheldon W. Prognosis in early adult life of coeliac children treated with a gluten free diet. Br Med J 1969;ii:401-4.

2 Young WF, Pringle EM. 110 children with coeliac disease 1950-1969. Arch Dis Child 1971;46:421-36.

${ }^{3}$ Mortimer PE, Stewart JS, Norman AP, Booth CC. Follow up study of coeliac disease. $\mathrm{Br}$ Med $J$ 1968;iii:7-9.

4 Jackson PT, Glasgow JFT, Thom R. Patients' understanding of coeliac disease and diet. Arch Dis Child 1985;60:672-4.

5 Weir DG, Hourihane O'B. Coeliac disease during the teenage period: the value of serial folate estimations. Gut 1974;15:450-7.

${ }^{6}$ Walker-Smith JA, Kilby A, France NE. Reinvestigation of children previously diagnosed as coeliac disease. In: McNicoll B, McCarthy CF, Fottrell PF, eds. Perspective in coeliac disease. Lancaster: MTP Press, 1978:451.

${ }^{7}$ Kumar PJ, O'Donoghue DP, Stenson K, Dawson AM. Reintroduction of gluten in adults and children with treated coeliac disease. Gut 1979;20:743-9.

${ }^{8}$ Visakorpi JK. Definition of coeliac disease in children. In: Hekkens W Th JM, Pena AS, eds. Coeliac disease. 2nd International Coeliac Symposium. Leiden: Sternfert-Kroese, 1974:

${ }^{9}$ Schmitz J, Arnaud-Battendier F, Jos J, Rey J. Long term followup of childhood coeliac disease (CD): is there a "natural recovery'? Pediatr Res 1984;18:1052.

10 Shmerling DH, Franckx J. Childhood celiac disease: a long term analysis of relapses in 91 patients. J Pediatr Gastroenterol Nutr 1986;5:565-9.

" Egan-Mitchell B, Fottrell PF, McNicholl B. Prolonged gluten intolerance in treated coeliac disease. In: McNicholl B, McCarthy CF, Fottrell PF, eds. Perspectives in coeliac disease. Lancaster: MTP Press, 1978;251-8.

Correspondence to Dr PJ Kumar, Department of Gastroenterology, St Bartholomew's Hospital, West Smithfield, London EC1A 7BE.

Accepted 2 March 1988 